FERMILAB-TM-2234

\title{
A Synchrotron Phase Detector for the Fermilab Booster
}

\author{
Xi Yang and Rene Padilla \\ Fermi National Accelerator Laboratory \\ Box 500, Batavia IL 60510
}

\begin{abstract}
A synchrotron phase detector is diagnostic tool for measuring the relative phase between the accelerating field and the beam. One has been implemented in the Fermilab Booster. This is probably the first time for the Booster that the accelerating voltage seen by the beam can be experimentally determined from the information of the synchrotron phase measurement and the existing total rf accelerating voltage (RFSUM) signal without using the calculated synchrotron phase values.
\end{abstract}

\section{Introduction}

A synchrotron phase detector is important for the purpose of directly measuring the phase relationship between the beam and the accelerating gap voltage at the $\mathrm{rf}$ cavities. It is a powerful diagnostic tool for examining the stability and efficiency of the feedback control system including the whole loop starting from low level rf (LLRF) driver to high level rf (HLRF) accelerating voltage. Besides that, the motivation of measuring the particle distribution in longitudinal phase space (LPS) at different times of an accelerating cycle and from these particle distributions to extract the relationship between the longitudinal emittance growth and the accelerating voltage (RFSUM) underlies our effort to build a synchrotron phase detector. The significance of the synchrotron phase detector is that before the synchrotron phase detector was implemented, the effective accelerating voltage seen by the beam only could be derived from the RFSUM signal and the calculated synchrotron phase. The synchrotron phase calculation requires that the longitudinal impedance be known. However with the synchrotron phase detector, one can obtain the synchrotron phase experimentally

\section{Design}

The synchrotron phase detector system includes two rf input signals, the phase detector [1], which has a bandwidth of at least $200 \mathrm{MHz}$ and uses a Log comparison of two rf signals, and a program to translate the direct phase measurement of two rf input signals 
into the synchrotron phase coordinate. Here, we specialize to the synchrotron phase detector in the Fermilab Booster, a rather typical example. However, there is a complication that is somewhat special. The rf reference signal must be summed from at least one cavity in each of two groups. These cavity groups, called Group A and Group $\mathrm{B}$, can be run with arbitrary but opposite time dependent offsets from the synchronous phase to control the effective rf amplitude acting on the beam. The rf signal from the beam is taken from the resistive wall monitor in the straight section long 18 of the Booster ring. The signal to noise ratio from the beam current pickup is improved by connecting it through a bandpass filter with a pass band of $29 \mathrm{MHz}$ to $63 \mathrm{MHz}$ because the $6 \mathrm{GHz}$ bandwidth of the resistive wall monitor passes much other information besides the phase of the rf fundamental component. The other input signal to the phase detector is the vector sum of two downstream gap pickups, from station 7 of Group A and station 8 of Group B. Cable lengths of the two input signals to the phase detector were carefully matched since Booster RF frequency changes from $38 \mathrm{MHz}$ at injection to $53 \mathrm{MHz}$ at extraction. The final adjustment of the two cable lengths was made using a criterion of zero degree synchrotron phase at injection. The phase detector delivers a signal of 9 degree per volt. The output of the phase detector is the relative phase between the beam signal and the effective RF voltage at the gap of RF cavities.

\section{Two Options for Forming the Sum Signal}

We ran into a difficulty when we tried to get the vector sum of two downstream gap pickups, one from station 8 of Group B and one from station 7 of Group A, to represent the $\mathrm{rf}$ voltage acting on the beam from these two stations. They are in the same long straight section 17 of the Booster ring. The beam has a constant of $152^{\circ}$ phase advance between them while the beam flight time changes because of the acceleration. The phase advance is determined by the ratio of the beam flight time to the rf period during the entire acceleration from $400 \mathrm{MeV}$ to $8 \mathrm{GeV}$ as calculated by Eq. 1 .

$$
\Delta \phi(t)=(L \times f(t) /(\beta(t) c)) \times 360^{\circ}
$$

Here, $L$ is the distance between these two stations, $f(t)$ is the rf frequency at time $t$ of a Booster cycle, $\beta(t) c$ is the velocity of the beam at time $t$. It is clear that the beam has a constant phase advance between these two stations if the ratio of the beam velocity and 
the $\mathrm{rf}$ frequency is kept constant. It is necessary to add a delay to the upstream gap pickup in such a way that it provides a constant of $152^{\circ}$ phase shift while the rf frequency changes from $38 \mathrm{MHz}$ to $53 \mathrm{MHz}$.

Our first approach was to use a cable delay to the pickup of the upstream rf station. Although it is impossible to use a cable delay to obtain a constant $152^{\circ}$ phase shift over the frequency range, one can correct the error introduced by the fixed cable delay. The cable length would need to be varied from $11.14 \mathrm{~ns}$ to $8.0 \mathrm{~ns}$ in order to maintain a $152^{\circ}$ phase shift, see Fig. 1(a). If an 11.14 ns cable is used to provide $152^{\circ}$ phase shift for the gap pickup of station 7 at injection, the phase error caused by the fixed cable length is shown as the red curve in Fig. 1(b). The phase error curve when an 8 ns cable is used for providing $152^{\circ}$ phase shift at extraction is shown as the black curve. A 9 ns cable is chosen according to Fig. 1(a) because we have a particular interest in the synchrotron phase measurement when RFSUM is reduced at $5.5 \mathrm{~ms}$ to $6.5 \mathrm{~ms}$ in the cycle.

The synchrotron phase measurement using the vector sum of two gap pickups from station 7 and station 8 with a 9 ns cable delay for the pickup of station 7 is shown in Fig. 2(a). The red and black curves represent the same synchrotron phase measurement before and after the correction. Here, the correction, shown in Fig. 2(b), is calculated from

Eq. 2.

$\left.\Delta(\Delta \phi)(t)=\left(\left(\left(152^{0} / 360^{\circ}\right) \times T_{R F}(t)\right)-9\right) / T_{R F}(t)\right) \times 360^{\circ}$

$T_{R F}(t)$ is the RF period at time $t$ of a Booster cycle. $\left(152^{\circ} / 360^{\circ}\right) T_{R F}(t)-9$ is the deviation of the beam flight time from station 7 to station 8 at time $t$ with fixed 9 ns cable delay. The delay is added to the gap pickup of station 7 before it is summed with the gap pickup of station 8 . The dispersion from the 9 ns cable can be neglected because the cable length is less than a half wavelength in the range of $38 \mathrm{MHz}$ to $53 \mathrm{MHz}$. The synchrotron phase measurement after correction, the black curve in Fig. 2(a), has the general features expected but some details worthy of investigation. 
A refinement to making the vector sum of the pickups represent the accelerating voltage seen by the beam is made by employing a wide band phase shifter providing $152^{\circ}$ phase advance to the downstream station 8 to subtract the phase delay introduced by the beam moving from station 7 to station 8 . The block diagram of the synchrotron phase detector with the wide band phase shifter is shown in Fig. 3. A 7 ns cable is removed from the signal of station 8 to compensate the time delay introduced by the wide band phase shifter. When the vector sum of pickups is taken as the reference rf input to the phase detector using the wide band phase shifter, the synchrotron phase measurement is as shown in Fig. 4. The measurement was made while the Booster intensity was $5.4 \times 10^{12}$ protons at extraction.

A final test to the synchrotron phase detector was to reduce the effective accelerating voltage and simultaneously observe the synchrotron phase change controlled by the feedback system. One expects that the synchrotron phase should move toward $90^{\circ}$ during the rf voltage reduction because of the phase feedback loop; the accelerating voltage is increased by making the synchrotron phase closer to $90^{\circ}$. Thus, the rf voltage reduction should be compensated by an increase in synchrotron phase. The result is shown in Fig. 5. The synchrotron phase of the beam did indeed approach $90^{\circ}$ during the rf voltage reduction.

\section{Potential Refinement}

Both correcting the synchrotron phase measurement when a $9 \mathrm{~ns}$ cable delay is added to the upstream pickup and using a wideband $152^{\circ}$ phase shifter added to the downstream pickup before the vector summation have the same small problem. The phase of the $\mathrm{rf}$ sum from station 7 and station 8 need not be exactly the same as the phase of RFSUM from all $\mathrm{rf}$ stations, primarily because each rf station has its own local feedback loop to compensate beam loading by detuning the cavity. Beam loading is detected by a phase detector measuring the phase difference between the rf drive to the power amplifier (PA) and the gap pickup signal from the rf cavity. The cavity is tuned to resonance with the drive through the control of the bias current in the tuner. The amount of the cavity detuning is determined by the phase error signal, which is developed by the beam induced phase shift at the gap pickup. Cable lengths of the rf drive and the gap pickup signal are carefully matched, so the phase difference between the drive and the gap 
pickup should be zero when no beam is present. The rf cavity output can be understood as the superposition of two sine waves, one from the $\mathrm{rf}$ field in the cavity without beam present and one from the beam induced wake field at the beam frequency. The sum is also a sine wave with a phase shift with respect to the zero beam rf field derived from the phase and amplitude of the wake field. Different rf cavities may have different responses to the beam induced wake field due to effects, such as power amplifier degradation, ferrite tuner power supply problems, etc. If so, there will be phase differences among the 18 rf cavities. Since the RFSUM signal is used to estimate the total volts per turn seen by the beam [2], it is obtained so that cable lengths from downstream gap pickups of 18 Booster rf stations are carefully matched before they are summed. In addition, wide band phase shifters are used to provide compensation for beam transit time from station to station with constant phase shifts over the entire Booster cycle. Thus, the RFSUM signal from all $\mathrm{rf}$ stations is the preferable reference $\mathrm{rf}$ input to the synchrotron phase detector. This has yet to be implemented because of practical obstacles, which can, however, be overcome without disturbing the functioning low level system.

\section{Acknowledgements}

Special thanks should be given to Chuck Ankenbrandt. He used his expertise to give authors important suggestions in the process of building the synchrotron phase detector. James MacLachlan also spent a lot of time in helping authors understand the basic related to this work. Without Milorad Popovic's support, this work couldn't have been completed quickly.

\section{References}

[1] R. D. Padilla, and B. Scala, Fermilab / Accelerator Division / RF Department / HLRF Group Document. (2002)

[2] J. Steimel, Fermilab / Accelerator Division / RF Department / HLRF (FanBack / FanOut) Document. (1992) 


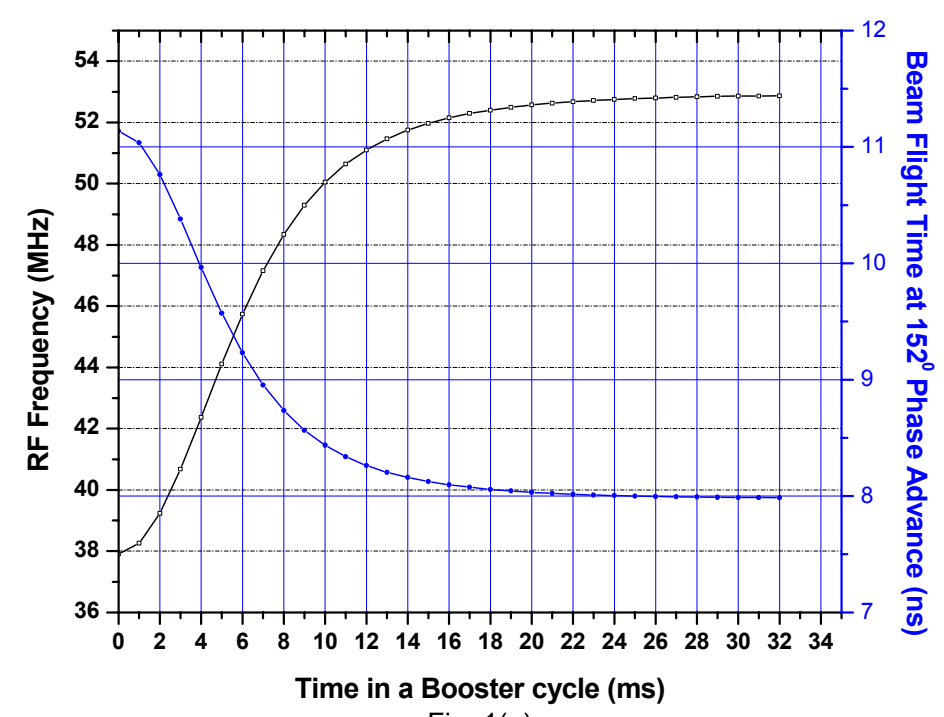

Fig. 1(a)

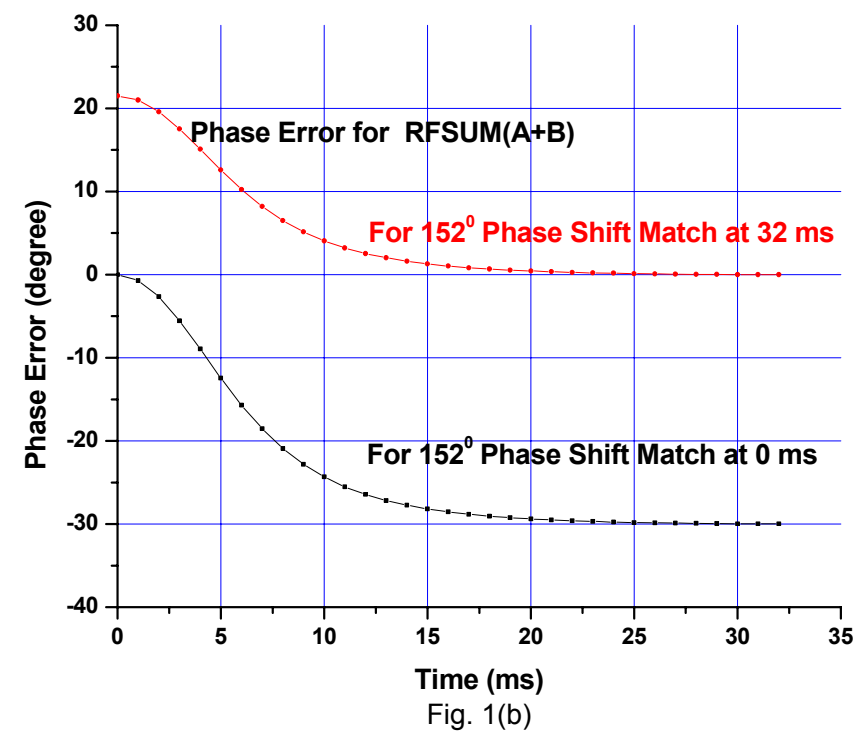

Figure 1(a) The black curve represents the Booster RF frequency vs. time in a Booster cycle, and the blue curve represents the flight time from station 7 to station 8 .

1(b) The red curve represents the phase error is introduced by adding an 8 ns cable to provide a $152^{\circ}$ phase shift in the Booster frequency range. The black curve represents the phase error introduced by adding a $11.14 \mathrm{~ns}$ cable to provide the phase shift. 


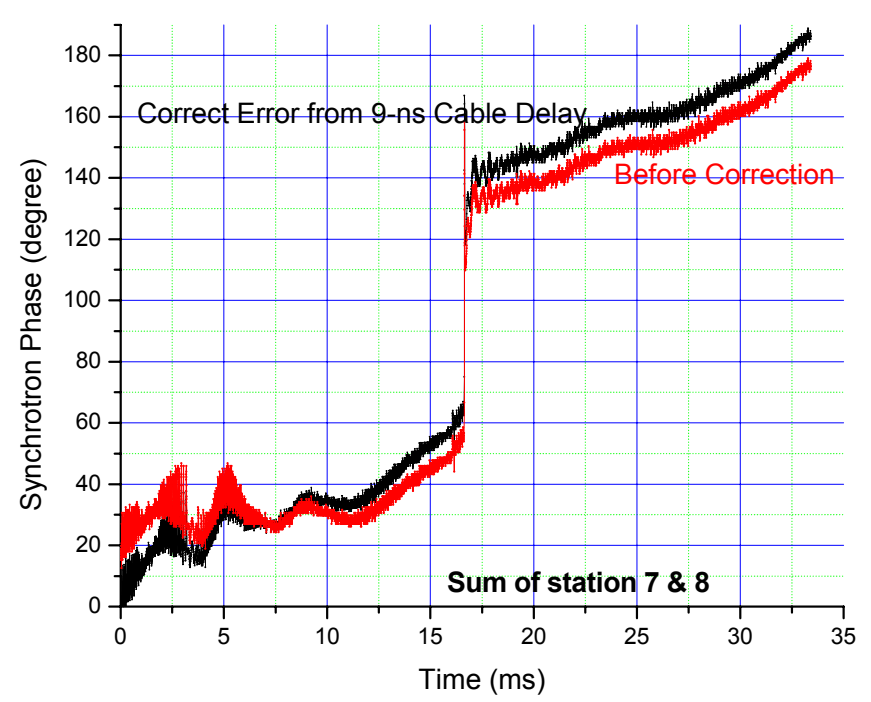

2(a)

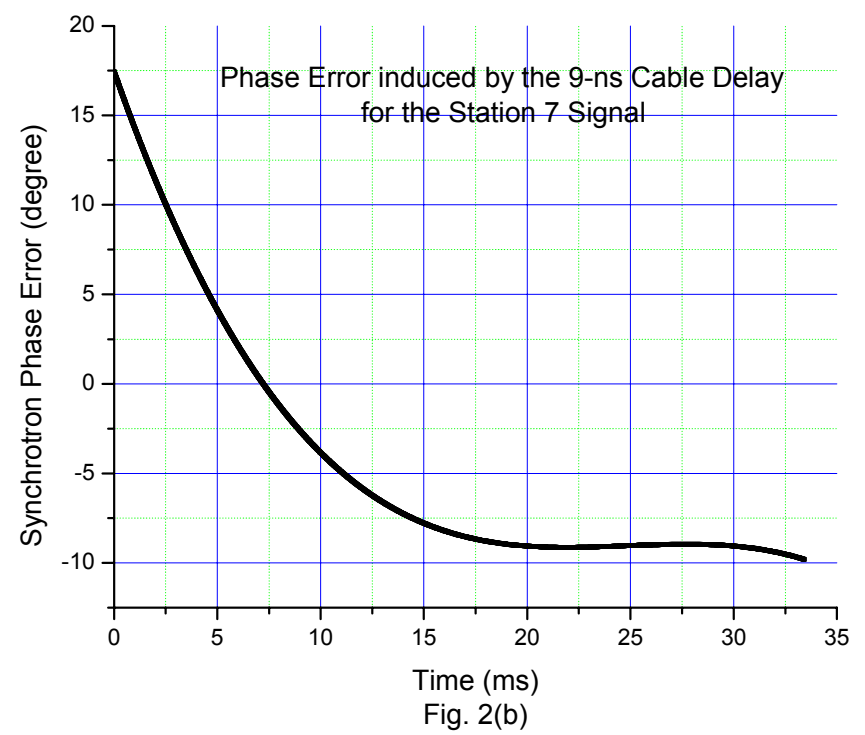

Figure 2(a) The red curve represents the synchrotron phase measurement when a 9 ns cable delay is used for the upstream pickup before it is summed with the downstream pickup. The black curve represents the synchrotron phase measurement with correction made by subtracting the error introduced by the cable delay.

2(b) The curve represents the phase error from a 9 ns delay cable $v s$. time in the cycle; it has been used to obtain the black curve in Fig. 2(a) from red curve in Fig. 2(a). 


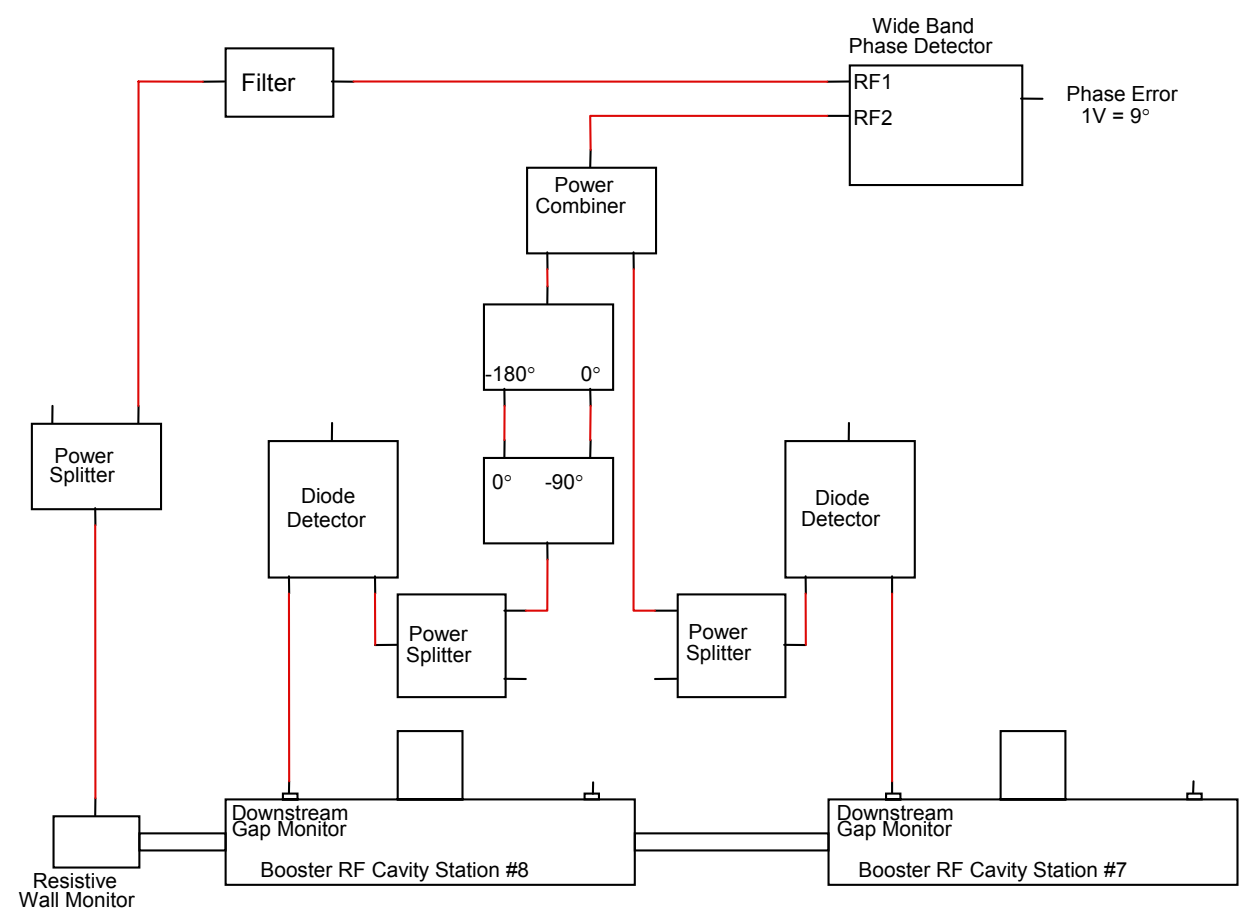

Figure 3 the block diagram shows the configuration of the synchrotron phase detector when the situation that a $152^{\circ}$ wideband shifter is added to the station 7 pickup before it is vector summed with the station 8 pickup. 


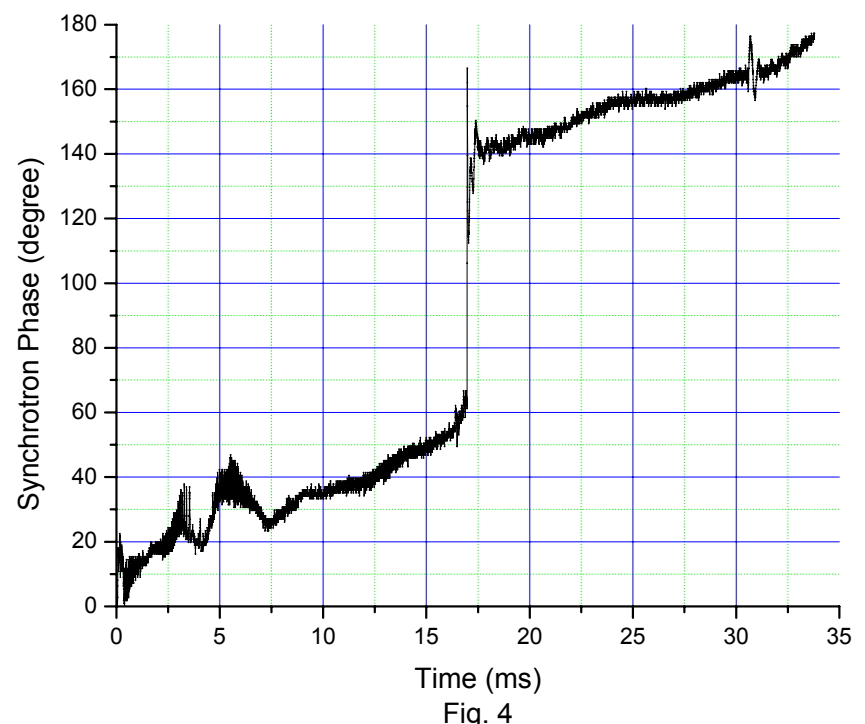

Fig. 4

Figure 4 The synchrotron phase $v s$. time in a Booster cycle using a wideband $152^{\circ}$ phase shifter in the station 8 pickup output. 

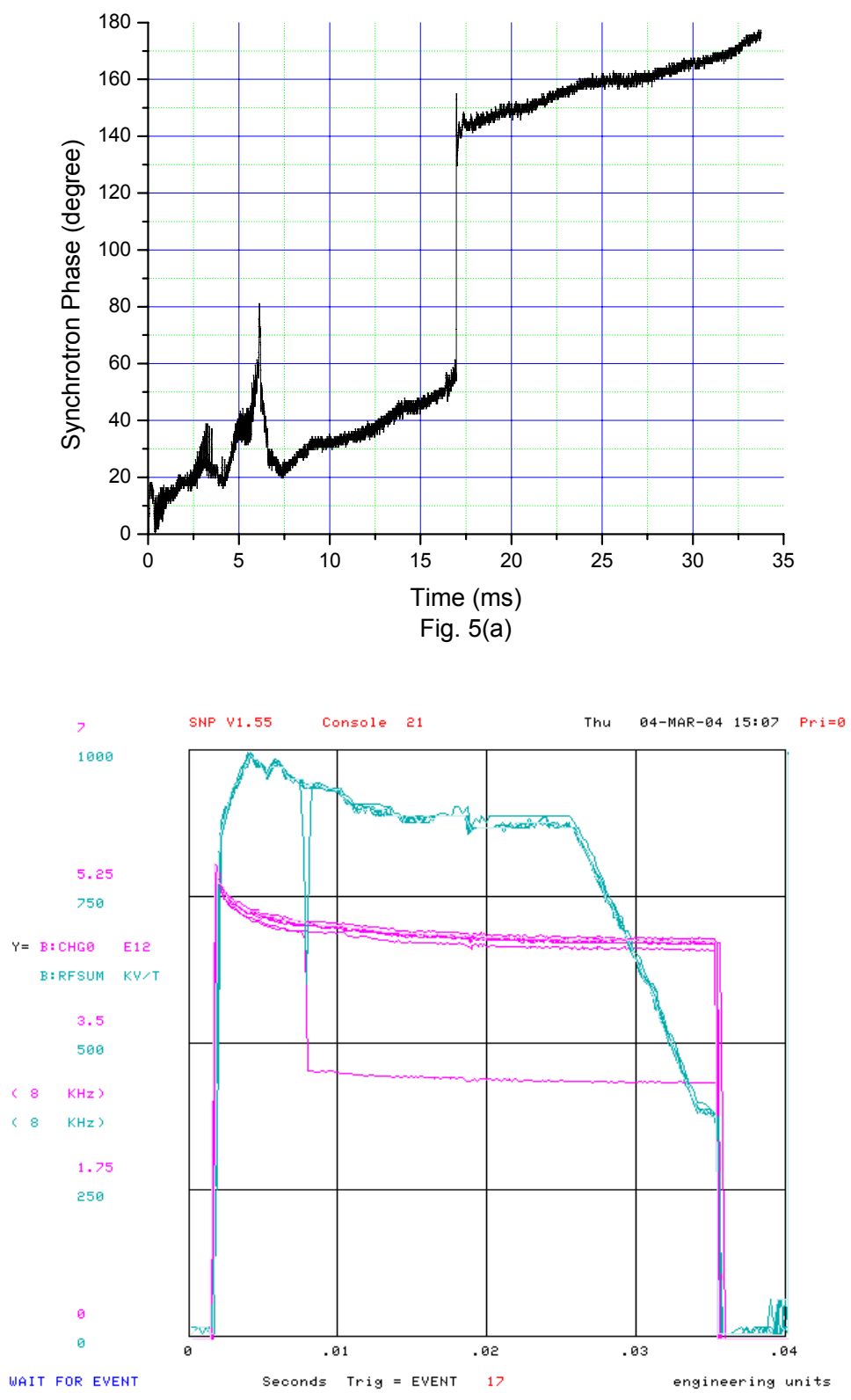

Figure 5(a) Synchrotron phase vs. time in a Booster cycle when RFSUM is reduced during the period 5.5-6.5 $\mathrm{ms}$ after injection. The synchrotron phase detector configuration is the same used in the measurement shown in Fig. 4.

5(b) Charge $v s$. time and RFSUM vs. time in the experimental condition of Fig. 5(a) 